International Journal of Wireless \& Mobile Networks (IJWMN) Vol. 4, No. 6, December 2012

\title{
TOPOLOGY MANAGEMENT IN WIRELESS SENSOR Networks: Multi-State Algorithms
}

\author{
Abrar Alajlan ${ }^{1}$, Benjamin Dasari ${ }^{2}$, Zyad Nossire $^{3}$, Khaled Elleithy ${ }^{4}$ and Varun \\ Pande $^{5}$ \\ ${ }^{1}$ Department of Computer Science and Engineering, University of Bridgeport, \\ Bridgeport, CT \\ aalajlanebridgeport .edu \\ ${ }^{2}$ Department of Computer Science and Engineering, University of Bridgeport, \\ Bridgeport, CT \\ bdasari@bridgeport. edu \\ ${ }^{3}$ Department of Computer Science and Engineering, University of Bridgeport, \\ Bridgeport, CT \\ znossire@bridgeport.edu \\ ${ }^{4}$ Department of Computer Science and Engineering, University of Bridgeport, \\ Bridgeport, CT \\ el leithy@bridgeport.edu \\ ${ }^{5}$ Department of Computer Science and Engineering, University of Bridgeport, \\ Bridgeport, CT \\ vpande@bridgeport. edu
}

\begin{abstract}
In order to maximize the network's lifetime and ensure the connectivity among the nodes, most topology management practices use a subgroup of nodes for routing. This paper provides an in-depth look at existing topology management control algorithms in Multi-state structure. We suggest a new algorithm based on Geographical Adaptive Fidelity (GAF) and Adaptive Self-Configuring Sensor Networks Topology (ASCENT). The new proposed algorithm outperforms both GAF and ASCENT algorithms.
\end{abstract}

\section{KEYWORDS}

Wireless Sensor Networks (WSN), Topology Management, GAF, ASCENT, Geographical Adaptive Fidelity, Adaptive Self-Configuring Sensor Networks Topologies, Topology control algorithm, Design Issues.

\section{INTRODUCTION}

Wireless sensor networks (WSNs) contain a large number of inexpensive sensor nodes deployed in divers environments, which used to detect data and deliver it to the sink. As Wireless Sensor Networks (WSNs) are becoming more and more popular because of its advantages and wide application range, research is growing intense in this area [1].

The advantage of being able to place remote sensing nodes without having to run wires and the cost related to it is a huge gain. And as the size of the circuitry of WSN is growing smaller along with the cost, the chances of their field of applications are growing large. In topology management of Wireless sensor network, energy efficiency is the primary factor. Most sensors depending on the requirement are battery powered and hence conserving the energy of these sensors is very crucial [2]. One straightforward approach is to turn off the radio of the sensor, thereby conserving energy. However, it is also important that a node must function to receive packets directed to it and help in other high level routing and control protocols. In a power conserving topology control algorithm for multi-hop ad-hoc WSN, we save power through

DOI : 10.5121/ijwmn.2012.4602 
International Journal of Wireless \& Mobile Networks (IJWMN) Vol. 4, No. 6, December 2012

routing coordination. There should be a minimum delay between nodes forwarding packets from source to destination than if all nodes being awake and the non-participating nodes should have radios turned off. The backbone is reflected by the paths that can function without interference [3].

In a network, it is not always productive to have every node with maximum power transmission having the energy constraint property. Topology control is used in wireless sensor networks to ensure that there are sufficient activated nodes connected with each other in order to deliver the data to the sink while the needless nodes are turning off to reduce energy consumption [3][4].

The topology control protocol allows each node to regulate its transmission energy. Each node should be able to control its transmission power. However, despite having the transmission power control, this should not disturb the node's connectivity i.e., the algorithm should make sure that adjacent nodes are reached which are immediately linked within high transmission power range [6].

We have seen that network topology is the basis for other protocol implementation and is the first step for designing and setting up a network. There are several aspects like data fusion, time synchronization, object location, etc. for which a potential topology is the basis and helps better the efficiency of routing and MAC protocol. The current research on WSN topology focuses mainly on the study of topology control to set up an optimized network for data transfer on the premise of meeting the requirements of network coverage and connectivity by controlling power, selecting backbone node and eliminating unnecessary communication link [7].

The WSN network could be star, cluster, mesh, or hybrid topology [8]. The first network applied in Wireless Sensor Network was the star network, which was a single-hop wireless sensor network and had the characteristics of low power consumption and simple structure. However, the disadvantage was that the whole network could be rendered non-functional if the central node were attacked, which was the bottleneck of the network [9]. Next in line was the cluster network, which is a multi-cluster network with cluster heads. Each cluster head receives data from their respective clusters and then forward this data to the base station after aggregating the data. The cluster network gave way to quite a number of algorithms, which used this design. The next was the mesh network with the ability of better fault tolerance. In this networks all nodes are equal in the sense that they can transmit to any neighbour node and then forward to the base station [10]. Apart from the ones mentioned above, there are also hybrid networks, which are a combination of two or three single topology together. Now a day, researchers of topology control focus on mesh networks because of its reliability factor, which provide multiple paths between two nodes. Different data transmission routes could improve the network connectivity. However, multiple paths architectures have high power consumption [7].

A primary research direction in Wireless Sensor Networks topology control is power control and sleep scheduling. The transmit power selection is power control and the changing of nodes between active and sleep modes so as to gain energy and have good topology is sleep scheduling. The main target of research in WSNs is to decrease the power consumption and increase the network lifetime since the energy consumption is proportional to distance in the power of communication [7].

The paper is structured as follows. Section 2 identifies the problem of wireless sensor networks regarding energy consumption. Section 3 provides an overview the related work of topology management and examines two topology protocols. Section 4 defines the proposed algorithm. Section 5 describes the simulation setup. Finally, section 6 concludes the paper on the basis of findings. 
International Journal of Wireless \& Mobile Networks (IJWMN) Vol. 4, No. 6, December 2012

\section{PROBLEM IDENTIFICATION}

The total lifetime of a battery-powered wireless sensor network is limited by the battery's capacity. Since disposable batteries are the main source of energy for most WSN's, there are two important hurdles in using them, which are the maintenance issues in rough areas and the scalability of the network. Therefore, most of the sensors get their source of energy from their internal limited powered battery and it cannot be re-powered after the battery is run dry due to its inaccessibility factor, overhead cost, etc. In order to increase the operational lifetime, we provides in this paper an algorithms technique that can be used to maximize the lifetime as much as possible while ensuring connectivity among the nodes.

In this paper we focus on GAF algorithm, which is energy saving algorithm. We present an efficient mechanism than GAF. GAF's mechanism of translation between states helps conserve quite a lot of energy. The mechanism of dividing the area into virtual grids is support clustering. However, GAF cannot reach its best level of conserving energy as it selects the cluster head arbitrarily without bearing in mind the best location for the head node.

\section{RELATED WORK}

The nodes are divided into equivalence classes based on the geographical properties in a lot of topology management algorithms such as GAF, DGAF, ASCENT, etc. Each class has its own representative node and only these turning their radio transmitters $\mathrm{ON}$ and carry out the routing functions thereby conserving energy. The remaining nodes either turn their radios OFF or enter into sleep mode depending on the algorithm.

The GAF algorithm which is based on energy model takes also into consideration the energy consumed in idle time which is also relatively high and hence proposes energy optimization by turning the radio of the sensor node OFF [11].

\subsection{Geographical Adaptive Fidelity (GAF)}

GAF stands for Geographical Adaptive Fidelity. This algorithm helps to decrease the energy consumption by $60 \%$ than an unaltered ad hoc routing algorithm and also the life of the network increases consistently to the density of nodes. Only one node from each grid is selected to be awake while the other are remain a sleep.

A state changeover diagram is shown in fig1.where a node initially starts in the discovery state where its turn its radio no and able to communicate and exchange discovery messages. The discovery message consists of node identification number, grid identification number, estimated active time and node state. In discovery state, a node sets up a timer T-discovery and when this timer expires, the node moves into active state. In active state, the node set a timer T-active to inform other nodes about how long this node will be in active state. After T-active expires, the node moves to discovery state again. A node in discovery or active state can moves into sleep if there is other nodes can perform the routing functions. In sleep state, sets a timer T-sleep and turn off its radio and moves into discovery when the timer expires [12].

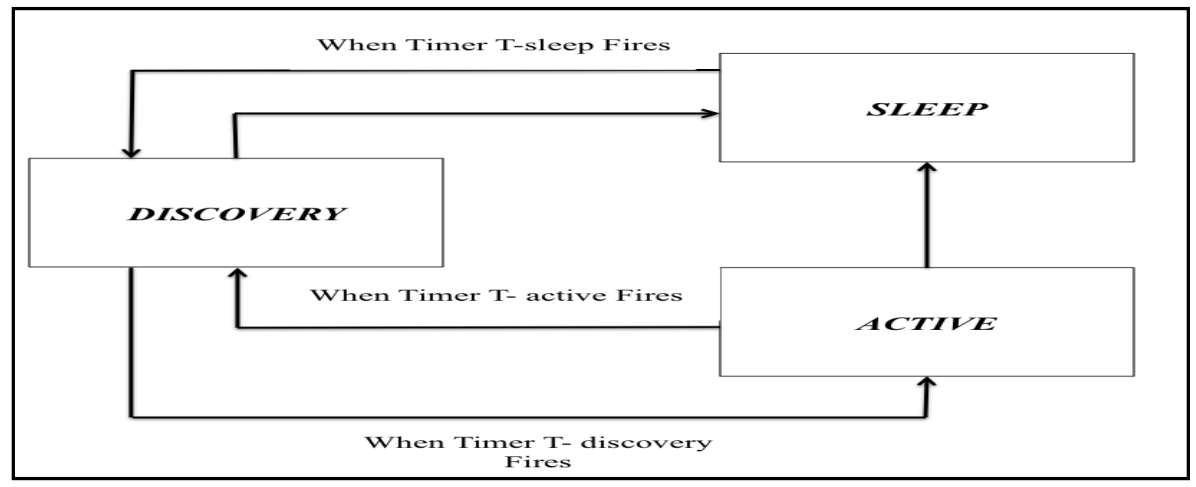

Figure 1: State changeover of a node in GAF protocol 
International Journal of Wireless \& Mobile Networks (IJWMN) Vol. 4, No. 6, December 2012

As the mass of a network employing GAF increases, the number of nodes in each grid is increases whereas the number of activated nodes remains the same [4]. However, a GPS is mandatory for this procedure. GAF splits the complete region into small virtual grids. The size of virtual grid is determined based on the maximum radio transmission power $\mathrm{R}$ [6]. Each grid is in such a way that for 2 neighbouring grids $\mathrm{X}$ and $\mathrm{Y}$, all nodes in $\mathrm{X}$ can interact with nodes in $\mathrm{Y}$ and all nodes in $\mathrm{Y}$ can interact with nodes in $\mathrm{X}$ as well. In GAF each node uses the information related to location using Global Positioning System and grid size to link with a virtual grid. From routing viewpoint, each node in a certain grid is equivalent. Also, it uses a load balancing technique to periodically waking up and switching in order to ensure all nodes keep functioning for maximum time. GAF has 2 assumptions:

(I) Node's communication range is deterministic.

(II) Exact node position is known.

Based on the above approach, the nodes can either be in sleep, discovery or active states. To start with, all nodes begin in discovery state and individually turn their radio ON and exchange discovery messages. Discovery messages have information like node id, grid id, estimated node active time (ENAT), and the node state. A node keeps a timer Td when it goes into discovery state. After timer Td completes, it transmits its discovery message and goes into active state. In the active state, it has a break-time value Ta to decide the duration of stay in active state and then return to discovery state. A node in either discovery or active states passes to sleep mode when the node has made sure that another node will take care of the routing task. Once it enters sleep mode, it terminates, sets all timers and turns off its radio and only awakens post application dependent sleep time $\mathrm{T}_{\mathrm{s}}[12]$.

\subsubsection{Mean Square Error in GAF}

An MSE of zero, meaning that the estimator $\hat{\theta}$ predicts observations of the parameter $\theta$ with perfect accuracy, is the ideal, but is practically never possible[13].

The MSE is use in our network simulation for a study purpose for node networks ranging from a few hundred to thousands. Because we have two set of node s once that are active and the other set that is asleep. We use the MSEs as a measure of how well they explain the communication in a given set of parameters. The simulation model with the MSE is generally explaining the variability in the observations and is called the best-unbiased estimator or MVUE (Minimum Variance Unbiased Estimator).

A linear regression techniques called the analysis of variance estimate is used as the MSE for the part of the analysis and use the estimated MSE to determine the statistical significance of the nodes and their parameters like the distance, signal strength and geo location. The goal of this experimental simulation is to construct experiments in such a way that when the observations of multiple node structures are analyzed, we can get the MSE is close to zero and define a relative to the magnitude of at least one of the master node.

\subsection{Adaptive Self-Configuring Sensor Networks Topologies (ASCENT)}

This algorithm is based on connectivity and uses local connectivity measures. It turns the nodes ON/OFF depending on neighbour and packet loss threshold. When a node figures high data loss, it signals the other nodes to join the network to forward the messages. However if high data loss is due to collision then the node would reduce its duty cycle. A node only joins the networks if it is helpful. It uses self-configuring and adaptive techniques to conserve energy and increase network lifetime. There are four states that a node can be active in, that is sleep, passive, test and active. Once a node is elected to be active adaptively, all the other nodes are turned off and they frequently find out if they have to become active or not [12][14].

A node initially starts in test state and sets up a timer T- test. When T-test fires, the node enter to active state and able to routing data and exchanging messages with other active nodes. The number of active nodes shouldn't exceed the neighbour threshold. Thus, if the number of 
neighbour nodes in active states is greater than neighbour threshold before T-test fires, the node enters to passive state where the node only able to listen to other active nodes without exchanging any messages. The idea behind the passive state is to collect information about the network with no congestion. In passive state, a node sets up a timer T-passive and when it expires the node moves into sleep state where the node turn off its radio signals and sets up timer T-sleep. When T-sleep expires, the node enters to passive state again [14]. Fig1. Illustrate the state changeover in ASCENT protocol.

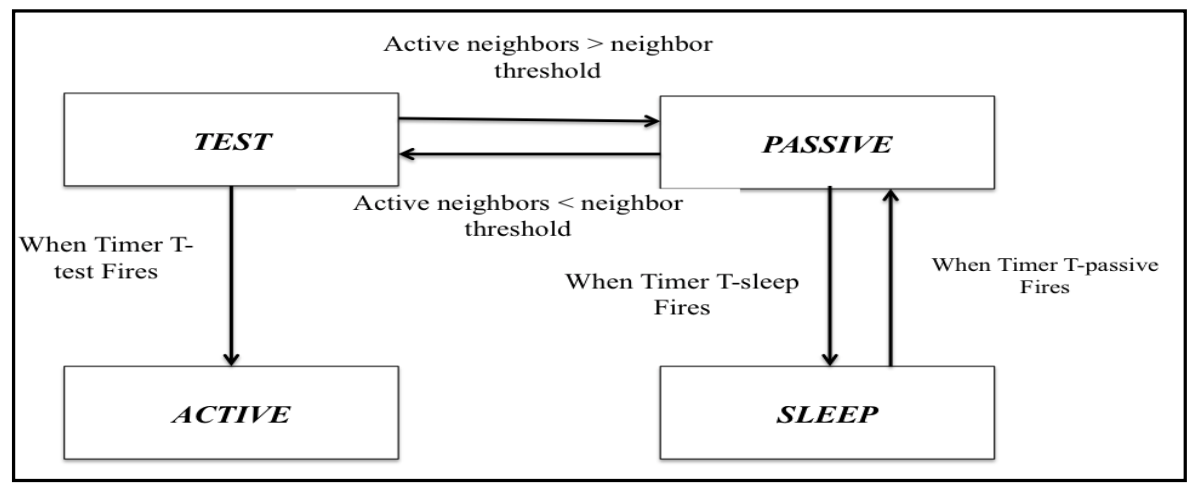

Figure 1: State changeover of a node in ASCENT protocol

A spread out topology control algorithm for varied WSN's was proposed previous research by bearing in mind irregular links. "They determine the existence of links among nodes by achieving the location of each vicinity node and exploiting radio propagation model" [7]. It is not suitable to design topology control structure minus the diminishing effect of radio signal into picture. The energy consumption for sensor node implementing topology control in simplified model is

$\mathrm{Ei}=\mathrm{Ti} \cdot \sum \mathrm{ni} \rightarrow$ for any node $\mathrm{i}$

In (1), Ei is the total power consumption of Node $\mathrm{i}$ during any time period, $\Delta \mathrm{t}$. Ti is the transmission power of Node $i$, and ni is the network traffic from Node i during any time period, $\Delta \mathrm{t}$

$\left(\mathrm{T}_{\max }-\mathrm{T}_{\min }\right) .\left(\sum \mathrm{ni} \rightarrow\right)$ for any node $\mathrm{i}$

(2) reflects the decreased quantity of energy consumption of Node i during the course of time, $\Delta t$, when it decreases broadcasting energy from $T_{\max }$ to ${ }_{T \text { min. }}$. The complete broadcast energy of a sensor node is $\mathrm{T}_{\max }$. The minimum broadcast energy that a sensor node must give in order to make sure of its link with its neighbouring nodes is $\mathrm{T}_{\min }$ [5].

Power control and sleeping scheduling are the main fields for the research on wireless sensor network topology control at present. Power control is the selection of transmits power and sleeping scheduling is the conversion of nodes between working and sleeping in order to obtain good topology and save energy [7].

Generally energy consumption of communication is proportional to distance in the power in WSN. As the distance increase, the energy consumption increases rapidly, and then the communication range is restricted for the limited energy of nodes [7].

\section{PROPOSED SOLUTION}

The main objective of this paper is to increase the network lifetime and conserve energy.

Although there are many algorithms, which focus on conserving energy and improve the network lifetime, we would be working on Improving GAF for better results. Although GAF helps saving considerable amount of energy, it selects the node head in a virtual grid quite randomly, which does not reach its best levels of conserving energy. In this paper, we focus as a part of proposed solution on the exact location of the node head in a virtual grid so as to 
increase the network lifetime when compared to the traditional GAF. When the cluster head node is chosen randomly, it might not be the best or most efficient node in that particular grid to get an efficient algorithm output. Therefore, studying the location of the cluster head node and deciding as to which node in the grid should be chosen for a specific field might lead us to new outputs. Fig 3. below lustrated the flow chart of the proposed algorithm.

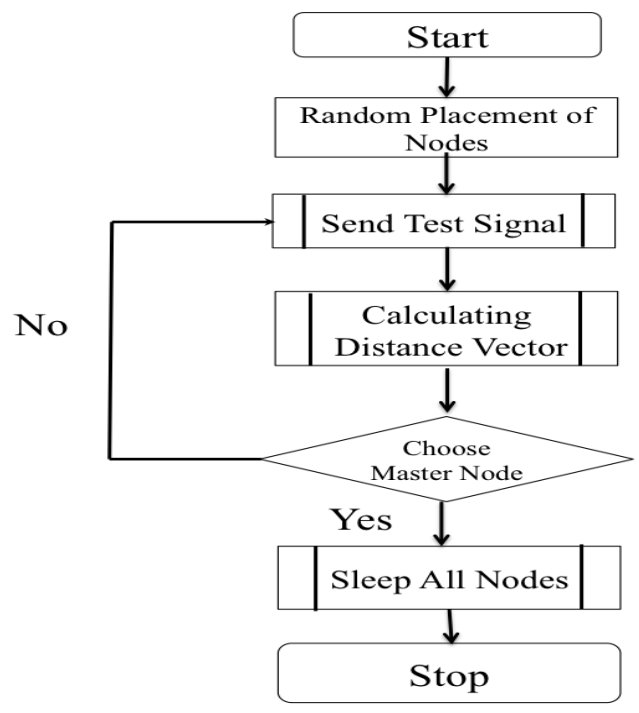

Figure 3: A Flow Chart of Proposed Algorithm

The proposed algorithm consists of the following steps:

1. Have a grid parameter setup for defining network location

2. Distribute all nodes in a network randomly

3. Send out a test signal

4. Calculate peer to peer node distance between all nodes in a single grid

5. Choose optimal node (auto selection based on the test signal strength and the distance vector)

6. Sleep states for all other nodes

The energy consumption of sending and receiving data are calculated by the formula:

$\mathrm{E}_{\text {send }}=\mathrm{k} \times \mathrm{E}_{\text {static }}+\mathrm{k} \times \varepsilon_{\text {amp }} \times \mathrm{d}^{2}$ and

$\mathrm{E}_{\text {receive }}=\mathrm{k} \times \mathrm{E}_{\text {static }}$ where

$\varepsilon_{\text {amp }}$ is the magnification of signal generator, $E_{\text {static }}$ is the energy consumption of the send and receive circuit and $\mathrm{d}$ is the transmission distance.

The number of packets sent by each node is equal and the location of the base station and the nodes in the network is constant. Distance between centre and the head node can be calculated as shown below:

$$
\begin{aligned}
& a=\sqrt{r^{2}-2 \cos \theta \times r \times l+l^{2}} \\
& \mathrm{E}_{\text {send }}=\mathrm{k} \times \mathrm{E}_{\text {static }}+\mathrm{k} \times \varepsilon_{\text {amp }} \times\left(\mathrm{r}^{2}-\theta \times \mathrm{r} \times l+l^{2}\right) \\
& \overline{\mathrm{E}}=\sum_{\substack{\Delta \mathrm{r} \rightarrow 0 \\
\Delta \theta \rightarrow 0}}^{\rho} \times \Delta \mathrm{r} \times \mathrm{r} \Delta \theta \times\left[\mathrm{k} \times \mathrm{E}_{\text {static }}+\mathrm{k} \times \varepsilon_{\mathrm{amp}} \times\left(\mathrm{r}^{2}-\theta \times \mathrm{r} \times l+l^{2}\right)\right]
\end{aligned}
$$


International Journal of Wireless \& Mobile Networks (IJWMN) Vol. 4, No. 6, December 2012

The expectation of the consumption can be inferred by the above formula.

$$
\begin{gathered}
=\rho k \times \int_{0}^{r^{2 \pi}} \int_{0}^{R}\left[\mathrm{E}_{\text {static }}+\mathrm{k} \times \varepsilon_{\text {amp }} \times(\mathrm{r} 2-\theta \times \mathrm{r} \times l+l 2)\right] r d r d \theta \\
\rho k \mathrm{E}_{\text {static }} \pi \mathrm{R}^{2}+\frac{\rho k \varepsilon_{\text {amp }} \pi}{2} \times \mathrm{R}^{2}+\rho k \varepsilon_{\text {amp }} \pi \mathrm{R}^{2} l^{2}
\end{gathered}
$$

When we place the values for the above parameters, we infer that we save twice as much energy when the head node is selected at the centre than otherwise.

\section{SIMULATION RESULTS}

In this simulation, we are going to explain how GAF helps us classify nodes based on radius called genetic algorithm radius. Consider that we have nodes, which are a grouping of diverse node groups from regions with similar nodes. This means that, although each node group has their own radius, the difference is only between radius parameters.

The graph below reflects a number of points on the X, Y coordinate. Clearly our nodes are a combination of two node groups. We look at a condition that we already know the formula and each group is generated by $\mathrm{a}=\mathrm{y}=\mathrm{x}+\mathrm{b}$ radius. In this example, we do not have a grave situation in the classification while there are only two groups and nodes are separated clearly. If the difficulty of the problem increases exponentially then we cannot monitor such processes. This would consume billions of mathematical calculations and therefore we need genetic algorithm and had to select the model of GAF.

Below is our simulation of GAF where all the nodes initially in sleep state and placed in the $\mathrm{XY}$-axis randomly and as shown in fig 4 . Then we select a particular grid consisting of $\mathrm{n}$ nodes and all of them would be in inactive state in the beginning. We then send a test signal to all the nodes to make sure that all the nodes are working as seen in fig 5. Finally, as seen in fig. 6, the head node is selected at the centre of the grid and all the other nodes are turned off conserving energy.

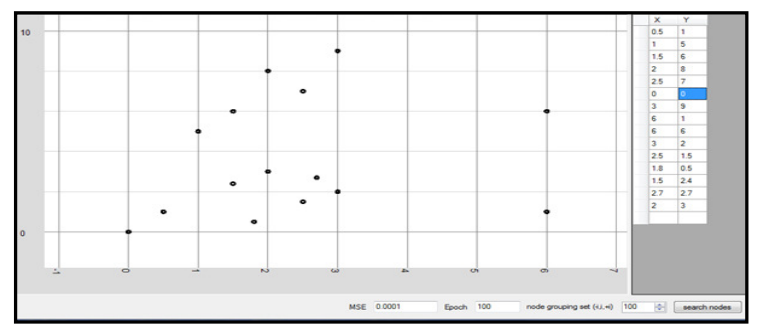

Figure 4: The simulation of GAF where all the nodes are placed in the XY-axis randomly.

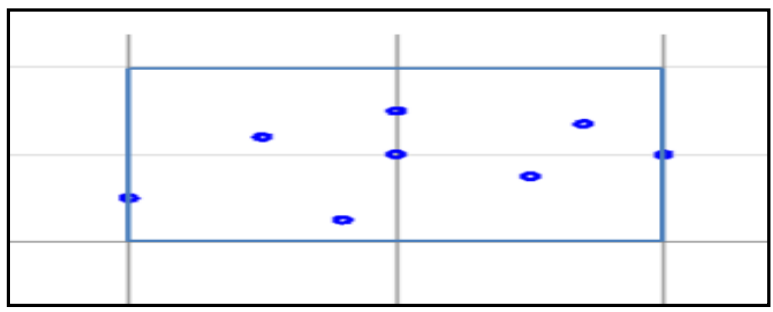

Figure 5: A particular grid consisting of $n$ nodes and all of them would be in inactive state in the beginning. 


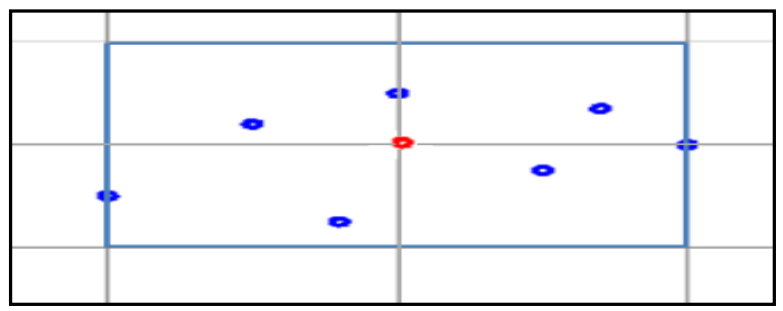

Figure 6: the head node is selected at the centre of the grid and all the other nodes are turned off conserving energy.

Our simulation is simply based on classifying diverse nodes centred on their obtainable connection. Consider an array of nodes group indexes. This array has a length equal to $\mathrm{k}$ nodes and each node has X and Y co-ordinates. From fig. 6, we can have an array of 0's and 1's since we considered only 2 node groups. The array can follow changing forms with its own mean squared error.

\begin{tabular}{|l|l|l|l|l|l|l|l|l|l|l|l|l|}
\hline 0 & 0 & 1 & 1 & 0 & 1 & 0 & 0 & 1 & 0 & 1 & 1 & 1 \\
\hline
\end{tabular}

Figure 7: Ten diverse arrangements of such an array of 0's and 1's.

\section{CONCLUSION}

Research must carry on in all the capacities of topology management such as sensing, data processing (computation) and communication. A field of probable research is to come up with an ideal assembling algorithm, which can be achieved when we combine the strong points of present working algorithms, working on removing the drawbacks and the suppositions. Though it might be seem like a herculean task, when we try to address the suppositions made by most algorithms we can come up with a reliable algorithm. The most significant assumption that should be taken care of is that of broadcasting data. Also the fresh algorithm must note the node failures in the Wireless Sensor Network, which includes the head node of the cluster too.

\section{REFERENCES}

[1] Rong Yu, Zhi Sun, and Shunliang Mei, "Scalable Topology and Energy Management in Wireless Sensor Networks", presented at the Wireless Communication and Networking Conference (WCNC) 2007.IEEE

[2] M. Tubaishat and S. Madria., Sensor Networks: an Overview., IEEE Potentials, 20-23, April 2003

[3] S. Roychowdhury and C. Patra, "Geographic Adaptive Fidelity and Geographic Energy Aware Routing in Ad Hoc Routing," International Conference, vol. 1, pp. 309-313, August 2010.

[4] Jawahar A, Radha S,” Energy Enhancement Using Hybrid Topology Management Scheme in Wireless Sensor Networks", presented at the India Conference (INDICON) 2009. IEEE

[5] Ya Xu, John Heidemann, and Deborah Estrin, “ Geography- Informed Energy Conservation for Ad Hoc Routing," in Proceedings of the Seventh Annual ACM/IEEE International Conference on Mobile Computing and Networking, 2001

[6] S. Hong., Y.-J. Choi., and S.-J. Kim., "An Energy Efficient Topology Control Protocol in Wireless Sensor Networks," presented at the International Conference on Advanced Communication Technology (ICACT) in IEEE, Feb, 2007. 
International Journal of Wireless \& Mobile Networks (IJWMN) Vol. 4, No. 6, December 2012

R. Yueqing. and X. Lixin., "A Study on Topological Characteristics of Wireless Sensor Network Based on Complex Network," presented at the International Conference on Computer Application and System Modeling (ICCASM) in IEEE, Beijing, China, 2010.

[8] S.Rickard, S.Svensson, T.Lennvall., "Energy Efficient Authentication in Wireless Sensor Networks," Emerging Technologies and Factory Automation (ETFA), 2007. IEEE

[9] Kay Romer and Friedemann Mattern.," The Design Space of Wireless Sensor Networks," presented at IEEE Wireless Communications, Vol. 11, No. 6, pp. 54-61Dec 2004.

[10] Hakan Bagci and Adnan Yazici.,"An Energy Aware Fuzzy Unequal Clustering Algorithm for Wireless Sensor Networks, "Fuzzy systems (FUZZ), presented at IEEE, July 2010

[11] J. You., D. Lieckfeldt., J. Salzmann., and D. Timmermann., "GAF\&Co: Connectivity Aware Topology Management for Sensor Networks," presented at the Institute of Applied Microelectronics and Computer Engineering in IEEE, Germany, 2009.

[12] Nedal Ababneh and S. Selvadurai, "Topology Control Algorithms for Wireless Sensor Networks: An Overview," International Journal on Wireless \& Optical Communications, vol. 3, pp. 49-68, 2006.

[13] T. Aktaran-Kalayc, D.Goldsman, C.Alexopoulos, J.Wilson, “ On The Mean-Squared Error Of Variance Estimators For Computer,” Winter Simulation Conference, IEEE 2011

[14] Alberto Cerpa and Deborah Estrin, "ASCENT: Adaptive Self-Configuring sEnsor Networks Topologies," presented at INFOCOM. Joint Conference of the IEEE and Communication Societies, 2002.

\section{Authors}

Mrs. Abrar Alajlan: is a Ph.D. student department of Computer Science and Engineering at the University of Bridgeport, Bridgeport, CT. She earned a master's degree in MBA with a concentration in Information Systems (IS) from Troy University, Troy, AL in 2011. She received a BS in Computer Science from Umm Al-Qura University, Makkah, Saudi Arabia. Abrar's interests are in Wireless Sensor Network (WSN), Network Security, Mobile Communication.

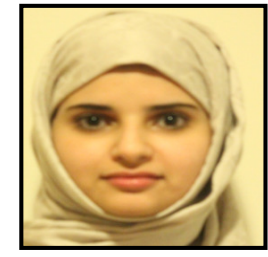

Mr. Benjamin Dasari: I am currently pursuing my Master of Science in Computer Science degree from University of Bridgeport. I have obtained and undergraduate degree in Mathematics, Electronics and Computer Science from Bhavan's Vivekananda Degree College in 2005 with a GPA of 3.80(approx.). Prior to my Master's, I worked with Automatic Data Processing (ADP) for five years in Hyderabad. I am interested in programming and researching new technologies.

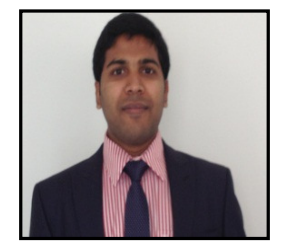

Mr. Zyad Nossire: received the B.Sc. in Management Information System from Al-Albyat University, Al-Mafrq ON, Jordan in 2006, and the MCA (Master of science information and Communication Technology ) from Utara University Malaysia in 2008. In 2012 Zyad Nossire joined University of Bridgeport as Ph.D. student in computer science and engineering at the University of Bridgeport, Connecticut-USA. From 2008-2009, Zyad Nossire was Assistant Lecturer in science and Technology Community College on Irbid - Al-Balqa Applied

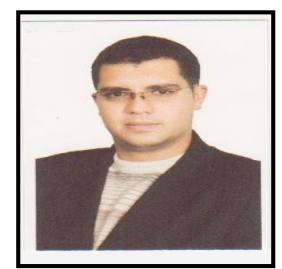
University-Jordan. From 2009 to 2011 Zyad Nossire joined Njran and Al-Emma Mohamed Ben Saoud University's -Saudi Arabia as assistant lecturer. Zyad Nossire research interest is in the general area of Cloud computing ,Mobile, wireless communications and networks. My email addresses: znossire@bridgeport.edu, ziad.jo2009@yahoo.com 
Dr. Khaled Elleithy: is the Associate Dean for Graduate Studies in the School of Engineering at the University of Bridgeport. His research interests are in the areas of, network security, mobile wireless communications formal approaches for design and verification and Mobile collaborative learning. He has published more than two hundreds research papers in international journals and conferences in his areas of expertise.

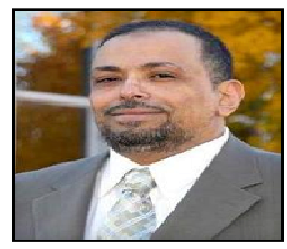

Dr. Elleithy is the co-chair of International Joint Conferences on Computer, Information, and Systems Sciences, and Engineering (CISSE).CISSE is the first Engineering/ Computing and Systems Research EConference in the world to be completely conducted online in real-time via the internet and was successfully running for four years. Dr. Elleithy is the editor or co-editor of 10 books published by Springer for advances on Innovations and Advanced Techniques in Systems, Computing Sciences and Software.

Dr. Elleithy received the B.Sc. degree in computer science and automatic control from Alexandria University in 1983, the MS Degree in computer networks from the same university in 1986, and the MS and Ph.D. degrees in computer science from The Center for Advanced Computer Studies in the University of Louisiana at Lafayette in 1988 and 1990, respectively. He received the award of "Distinguished Professor of the Year", University of Bridgeport, during the academic year 2006-2007.

Mr. Varun Pande is a Graduate Research Assistant currently attending the University of Bridgeport as a $\mathrm{PhD}$ candidate in Computer Science and Engineering. He graduated from the University of Bridgeport with a Master in computer Science in May of 2012. He had worked as a CSR representative at TATA Power, during his Bachelor in computer science and Information Technology. Currently and for the past two years he has been a Graduate Assistant and taught Labs on Wireless Sensor Communication using MICA z Motes. His research interests are Computer Vision, Image Processing, Parallel processing and Wireless Sensor Networks. He hopes to

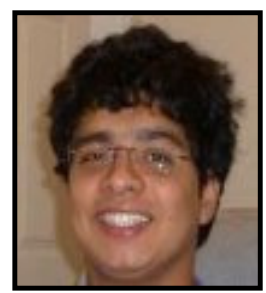
share my experiences, research and knowledge with other graduates and professionals to work in a collaborative research for a Better tomorrow! 